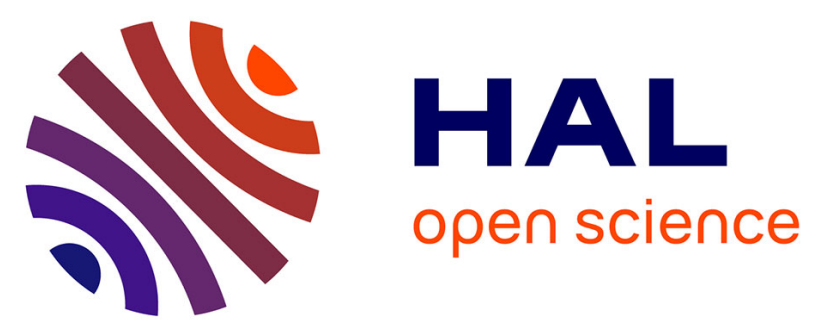

\title{
A new representation and projection model for tomography, based on separable B-splines
}

Fabien Momey, Loïc Denis, Catherine Mennessier, Éric Thiébaut, Jean-Marie Becker, Laurent Desbat

\section{- To cite this version:}

Fabien Momey, Loïc Denis, Catherine Mennessier, Éric Thiébaut, Jean-Marie Becker, et al.. A new representation and projection model for tomography, based on separable B-splines. 2011 IEEE Nuclear Science Symposium and Medical Imaging Conference, Oct 2011, Valencia, Spain. pp.MIC9.S-214. ujm-00670025

\section{HAL Id: ujm-00670025}

\section{https://hal-ujm.archives-ouvertes.fr/ujm-00670025}

Submitted on 14 Feb 2012

HAL is a multi-disciplinary open access archive for the deposit and dissemination of scientific research documents, whether they are published or not. The documents may come from teaching and research institutions in France or abroad, or from public or private research centers.
L'archive ouverte pluridisciplinaire HAL, est destinée au dépôt et à la diffusion de documents scientifiques de niveau recherche, publiés ou non, émanant des établissements d'enseignement et de recherche français ou étrangers, des laboratoires publics ou privés. 


\title{
A new representation and projection model for tomography, based on separable B-splines
}

\author{
Fabien Momey, Loïc Denis, Catherine Mennessier, Éric Thiébaut, Jean-Marie Becker, Laurent Desbat
}

\begin{abstract}
Data modelization in tomography is a key point for iterative reconstruction. The design of the projector, i.e. the numerical model of projection, is mostly influenced by the representation of the object of interest, decomposed on a discrete
\end{abstract} basis of functions.

Standard projector models are voxel or ray driven; more advanced models such as distance driven, use simple staircase voxels, giving rise to modelization errors due to their anisotropic behaviour. Moreover approximations made at the projection step amplify these errors. Though a more accurate projection could reduce approximation errors, characteristic functions of staircase voxels constitute a too coarse basis for representing a continuous function. As a result, pure modelization errors still hold. Spherically symmetric volume elements (blobs) have already been studied to eradicate such errors, but at the cost of increased complexity, because they require some tuning parameters for adapting them to this use.

We propose to use 3D B-splines, which are piecewise polynomials, as basis functions. When the degree of these polynomials is sufficiently high, they are very close from being with a spherical symmetry, i.e. blobs, avoiding projection inconsistencies, while keeping local influence and separability property. B-splines are considered, in sampling theory, as the almost optimal functions for the discretization of a continuous signal, not necessarily bandlimited, potentially allowing to reduce the angular sampling of the data without any loss of quality.

We show that the projection of $B$-splines can be approximated rather accurately by a separable function, independent from the angle of projection, easier to integrate on detector pixels. The higher the degree of the used B-splines, the better the quality of the approximation, but also the larger the number of required operations. Thanks to these approximations, a convenient tradeoff between the need of accuracy and a fast calculation can be obtained. This has resulted in the implementation of a more accurate numerical projector, which can deal with a reduced angular sampling without loss of performance. The additional computation cost is also efficiently reduced. We have studied the quality of enhancement involved by this projector on 2D iterative reconstructions of a Shepp-Logan phantom, from a small number of fan beam projections. Reconstructions have been performed by optimization methods, minimizing the squared data

Manuscript received November 22, 2011. This work was supported by the MiTiV project (Méthodes Inverses pour le Traitement en Imagerie du Vivant), funded by the French ANR ( ${ }^{\circ}$ ANR-09-EMER-008).

Fabien Momey is with the Centre de Recherche Astrophysique de Lyon Observatoire de Lyon, Lyon, France, and with the Laboratoire Hubert Curien, Université Jean Monnet, Saint-Étienne, France (telephone: +33 478868 546, e-mail: author fabien.momey@univ-lyon1.fr).

Loïc Denis is with the Laboratoire Hubert Curien, and has just left the Centre de Recherche Astrophysique de Lyon - Observatoire de Lyon.

Catherine Mennessier is with the Laboratoire Hubert Curien, and with the engineer school CPE, Lyon, France.

Éric Thiébaut is with the Centre de Recherche Astrophysique de Lyon Observatoire de Lyon.

Jean-Marie Becker is with the Laboratoire Hubert Curien, and with CPE Lyon.

Laurent Desbat is with the TIMC-IMAG, Grenoble, France. residuals with a regularization term, using an efficient QuasiNewton optimization algorithm.

\section{INTRODUCTION}

$\mathbf{I}$ TERATIVE reconstruction methods for tomography have long proven their potential to enhance reconstruction quality, compared to the filtered backprojection (FBP) [2]. The drawback of iterative methods is their expensive computation time. Due to very low signal-to-noise ratio in PET/SPECT imaging, iterative methods are preferred because they allow a better modelization of the underlying physics and counting statistic of positron annihilations. However FBP is still the method of choice in X-ray computed tomography. Ongoing researches on algorithms and recent enhancements in computational power, such as multi-core processor units or GPUbased implementation facilities, call for a re-evaluation of the potential of iterative reconstruction in this domain.

Such methods require a numerical modelization of the data acquisition process: the so-called projector. It is used for the reprojection of the current estimate of the image to be reconstructed, and compared with the true data at each iteration step. A backprojection operator is also needed, which is the transpose of this projector. This numerical model has to reproduce as accurately as possible the physical process of data acquisition, based on the mathematical principle of the $\mathrm{X}$-ray transform (Radon transform in 2D), while not increasing the computational burden.

The representation of the object of interest (image) is the starting point of the projector. The real nature of this image is a continuous signal, discretized for numerical purposes. Mathematically, it is assimilated to a continuous function decomposed on a discrete basis of functions. The choice of this basis is essential for an accurate representation of the true function. Standard models such as voxel driven or ray driven [5] are based on raw samples, linearly interpolated at the projection step, either in the image space or in the data space, yielding strong modelization errors and artifacts on the reconstructed image. Hence the quality of the modelization strongly depends on the sampling rate. More advanced models, such as the recent distance driven [1] projector, take a better account of the continuity of the image by using staircase voxels. These functions, uniform on their cubic support, are entirely projected on the detector plane. Their projection is calculated as the length of overlap of the projected support with the impinged detector pixels. Each voxel impinges at least one detector pixel for a given projection, and vice versa for the related backprojection. However such a basis of functions 
provides a coarse representation of the image. This kind of basis has an anisotropic behaviour, causing large modelization errors in the projector, and thus in the reconstruction step. Moreover, for implementation purposes, the projection of the staircase voxel, in the distance driven model, is approximated, increasing its modelization errors. In [3], a more accurate projection of the staircase voxel reduces these approximation errors, but errors due to this unadequate basis still hold. Such issues can be dealt with another type of basis functions, already considered: the spherically symmetric volume elements, mostly known as blobs [7] [8] [9] [10] [11]. Thanks to their sphericity property, the projection of such functions is totally isotropic (at least in parallel beam geometry), yielding a better modelization of the projection process. The most usual blobs are the Kaiser-Bessel functions. However, they require the tuning of many parameters to be used as a suitable basis for the representation of a continuous function. An apodization is necessary because their support is not compact, and optimal parameters have to be found to satisfy the partition of unity. A projector based on these blob representations is complex to handle.

We propose the use of B-splines as an alternative to both staircase voxel and blob approaches. B-splines are well known piecewise polynomial functions, and are characterized by the degree of their constituting polynomials. Recent works in sampling theory [17] [18] [14] have shown a large interest when such functions are used as a basis of representation of a continuous signal as a discrete sequence of coefficients, with a good recovering accuracy. Thus B-splines involve some of the best approximation order as their degree increases. The staircase voxel being in fact a B-spline of degree 0 , we turn to $\mathrm{B}$-splines of higher degree. Increasing their degree makes them more and more similar to the 3D Gaussian functions, with a quasi-isotropic behaviour, approximating quite well blobs main feature, while keeping local influence and separability property. All these properties indicate that B-splines would constitute a smart basis for image representation and projection modelization in tomography. This has already been done in [4] for 2D FBP reconstruction in parallel beam geometry, giving us clues to develop a new efficient numerical projector for iterative reconstruction. Moreover [4] shows that it is possible to reduce the angular sampling of projections without any loss of quality. We claim that it is also one of the most important improvements we might get with our B-spline-based projector approach.

The core of our projector lies in the way a tridimensional separable B-spline is projected on the detector plane. The exact projection, called footprint in the following, is approximated by a separable function on the detector, independently of the angle of projection, yielding an easier and faster integration on detector pixels. In section II, we detail some features of the basis of B-splines, then we present the principle of our projector, explaining our approximations and comparing them with the distance driven projector. In section III we present our iterative reconstruction scheme and show some results of $2 \mathrm{D}$ reconstructions, in fan beam geometry, of a SheppLogan phantom, with noiseless and noisy data, and from a few number of projections.

\section{Materials AND MethodS}

\section{A. General formalism of image representation}

Let $f: \boldsymbol{x} \mapsto f(\boldsymbol{x})$, with $\boldsymbol{x}=\left(x_{1}, x_{2}, \ldots, x_{n}\right) \in \mathbb{R}^{n}$, be the $n$-dimensional continuous function modelizing the true image to be reconstructed. Let $\tilde{f}: \boldsymbol{x} \mapsto \tilde{f}(\boldsymbol{x})$ be its approximate decomposition on a discrete basis of functions:

$$
\tilde{f}(\boldsymbol{x})=\sum_{\boldsymbol{k} \in \mathbb{Z}^{n}} \mathrm{c}_{\boldsymbol{k}} \varphi_{\boldsymbol{k}}(\boldsymbol{x})=\sum_{\boldsymbol{k} \in \mathbb{Z}^{n}} \mathrm{c}_{\boldsymbol{k}} \varphi\left(\boldsymbol{x}-\boldsymbol{x}_{\boldsymbol{k}}\right)
$$

where this discrete shift-invariant basis is assumed to be composed of the compact atom function $\varphi(\boldsymbol{x})$, regularly spaced on a $n$-dimensional grid of $N$ samples. $\boldsymbol{k}=\left(k_{1}, k_{2}, \ldots, k_{n}\right)^{\mathrm{T}} \in$ $\mathbb{Z}^{n}$ corresponds to indexes of the $N$ samples of the discrete grid in the $n$-dimensional space, $\boldsymbol{x}_{\boldsymbol{k}}=\left(x_{k_{1}}, x_{k_{2}}, \ldots, x_{k_{n}}\right)^{\mathrm{T}} \in$ $\mathbb{R}^{n}$ are the coordinates of this discrete grid.

The function $\tilde{f}$ is an approximation of $f$, where the $c_{k}$ coefficients must be determined so that the approximation error is minimal. Thus $\tilde{f}$ defines a continuous function from a discrete sequence of coefficients $c_{k}$. For numerical purposes, $\tilde{f}$ is described as a vector of its $N$ coefficients:

$$
\mathbf{c}=\left(\mathrm{c}_{\mathbf{1}}, \mathrm{c}_{\mathbf{2}}, \ldots, \mathrm{c}_{\boldsymbol{N}}\right)^{\mathrm{T}} \in \mathbb{R}^{n}
$$

Even though the basis functions $\varphi_{\boldsymbol{k}}$ have a compact support, they can spread over the neighboring samples (this is the case for B-splines of degree higher than 1). Thus, because $\varphi_{\boldsymbol{k}}(x) \geq$ 0 , the coefficients $c_{k}$ do not correspond to the samples values $\mathrm{f}_{\boldsymbol{k}}=\tilde{f}\left(\boldsymbol{x}_{\boldsymbol{k}}\right)$ :

$$
\mathbf{f}=\left(\mathrm{f}_{\mathbf{1}}, \mathrm{f}_{\mathbf{2}}, \ldots, \mathrm{f}_{\boldsymbol{N}}\right)^{\mathrm{T}}, \quad \text { with } \mathrm{f}_{\boldsymbol{k}}=\sum_{\boldsymbol{k}^{\prime} \in \mathbb{Z}^{n}} \mathrm{c}_{\boldsymbol{k}^{\prime}} \varphi_{\boldsymbol{k}^{\prime}}\left(\boldsymbol{x}_{\boldsymbol{k}}\right)
$$

Eq.(3) can be expressed as a matrix operator:

$$
\mathbf{f}=\mathbf{\Phi} \cdot \mathbf{c}
$$

where $\boldsymbol{\Phi}$ is, for the case of B-splines, the spline interpolation operator. Instead of solving a system of linear equations, there exists a very fast way to apply and to invert it, based on digital filtering [15] [16] [17]. Hence it is very easy to deal with either c or $\mathbf{f}$ in the reconstruction process, since the transformation from one space to another is simple to handle.

The choice of the atom function $\varphi$ of the basis is essential for warranting consistency with the image intrinsic continuity. It will be a key point for the design of the projector which has to modelize accurately the data. Desired properties are:

1. A good modelization of the continuity of the function, while preserving sharp edges: this property can be related to the approximation order of the basis;

2. A compact support, leading to a sparse projector;

3. Separability, allowing factorization of involved expressions, thus lowering the computational burden;

4. Robustness of the basis of functions with respect to artifacts generated by geometric transformations (rotation, registration, resampling);

5. Spherical symmetry for isotropic projection. 


\section{B. B-splines as basis functions}

Splines with degree $d$ are piecewise polynomial functions with degree at most $d$, continuously differentiable up to order $d-1$. Any spline can be written as a unique linear combination of regularly shifted atom piecewise polynomial functions. These atom functions are called B-splines [17].

Let

$$
\beta^{0}(x)= \begin{cases}1, & -\frac{1}{2}<x<\frac{1}{2} \\ \frac{1}{2}, & |x|=\frac{1}{2} \\ 0, & \text { otherwise }\end{cases}
$$

be the rectangular pulse. Let us now denote by $\beta^{d}$ a Bspline of degree $d$, constructed by $d+1$ convolutions of $\beta^{0}$, corresponding to the generic member of this family of functions.

$$
\beta^{d}(x)=\underbrace{\beta^{0} * \cdots * \beta^{0}}_{d+1 \text { terms }}(x)
$$

This is the atom we are going to consider. With this notation, a spline of degree $d$ can be written as follows:

$$
s(x)=\left(\sum_{k \in \mathbb{Z}} c_{k} \delta\left(x-x_{k}\right)\right) * \beta^{d}(x)=\sum_{k \in \mathbb{Z}} c_{k} \beta^{d}\left(x-x_{k}\right)
$$

where $\delta$ is the Dirac distribution. Hence going back to the formulation of the image representation in (1), we choose Bsplines as our basis of functions $\varphi$, leading to:

$$
\tilde{f}(\boldsymbol{x})=\sum_{\boldsymbol{k} \in \mathbb{Z}^{n}} \mathrm{c}_{\boldsymbol{k}} \boldsymbol{\beta}_{\boldsymbol{k}}^{d}(\boldsymbol{x})=\sum_{\boldsymbol{k} \in \mathbb{Z}^{n}} \mathrm{c}_{\boldsymbol{k}} \boldsymbol{\beta}^{d}\left(\boldsymbol{x}-\boldsymbol{x}_{\boldsymbol{k}}\right)
$$

where $\boldsymbol{\beta}^{d}$ is a $n$-dimensional B-spline of degree $d$, separable on each component of $\mathbb{R}^{n}$ :

$$
\boldsymbol{\beta}^{d}(\boldsymbol{x})=\prod_{l=1}^{n} \beta^{d}\left(x_{l}\right) .
$$

$\tilde{f}$ is thus a $n$-dimensional spline approximating the true function $f$. In spline sampling theory [17], the $\mathrm{c}_{\boldsymbol{k}}$ coefficients are chosen so that the approximation error $\|f-\tilde{f}\|_{L_{2}}$ is minimal, in the sense of $L_{2}$ norm. That is to say that $f$ is the orthogonal projection of $f$ on the space of splines of degree $d$, the functions $\boldsymbol{\beta}_{\boldsymbol{k}}^{d}$ being a basis of this space. As a result the $c_{k}$ are deduced from the $L_{2}$ inner product of $f$ with the dual of each shifted basis function $\boldsymbol{\beta}_{\boldsymbol{k}}^{d}$, denoted $\stackrel{\circ}{\boldsymbol{\beta}}_{\boldsymbol{k}}^{d}$ :

$$
\mathrm{c}_{\boldsymbol{k}}=\left\langle f(\boldsymbol{x}), \stackrel{\circ}{\boldsymbol{\beta}}_{\boldsymbol{k}}^{d}(\boldsymbol{x})\right\rangle
$$

Stated otherwise, the $\mathrm{c}_{\boldsymbol{k}}$ are the components of $\tilde{f}$ in the space defined by the basis of functions $\boldsymbol{\beta}_{\boldsymbol{k}}^{d}$.

For a 1-dimensional function, the error of approximation is $O\left(\Delta^{L}\right)$, where $L=d+1$ is the order of approximation and $\Delta$ is the sampling step [17] [18] [14]. Hence using Bsplines of higher degree decreases the approximation error, and potentially induces a reduction of the sampling step for a given tolerance.
As already mentioned in section I, classical basis functions used by some existing projectors are the simple staircase voxels, in other words B-splines of degree 0 . This is the case for the distance driven projector [1]. These functions are advantageous for being the most compact B-splines, easy to manipulate, with no spreading over the neighboring samples of the grid (as for the B-splines of degree 1). As a result we have $\mathbf{f}=\mathbf{c}$ in this case. Besides, staircase voxels suffer from a high anisotropic behaviour. In addition to their low approximation order, they constitute a too coarse basis of representation of a continuous object, leading to large modelization errors. A finer sampling rate lowers these errors, but at the cost of an increased computational burden.

The main goal of this paper is to show that the accuracy of the model can be improved using B-splines of higher degree. Indeed, B-splines being $d+1$-fold convolutions of a rectangular pulse, they are close to a Gaussian function when their degree $d$ is large, according to the central limit theorem. Thus they tend to spherically symmetric function, while preserving a local support. As a result we can deal with quasi-isotropic functions, at the expense of only a slight spreading of the support of these functions. We also get a better approximation order. These two properties are related by the fact that B-splines are the shortest and smoothest scaling functions for a given order of approximation [18]. Moreover, the cubic (degree 3) B-splines are members of the family of Moms functions (Maximum order minimum support), giving them a kind of optimality in this context [18].

The use of blobs, for instance the Kaiser-Bessel functions, has also improved the quality of image modelization, due to their isotropic behaviour [7] [8] [9] [10] [11], giving a more accurate projection on the detector. However, as stated in section I, they have to be tuned to comply with sampling theory, for example the partition of unity. Thus many parameters need to be adjusted to approach optimal performances. Moreover, because these functions are not separable, the computation of a blob-based projector is more expensive, or needs approximations to accelerate the calculation. These observations have convinced us that this basis is too complex and therefore is not adapted enough to our problem, unlike B-splines. However the comparison of our approach with blobs is not in the scope of this paper, and we are aware that a thorough study is necessary before concluding this subject.

\section{Projector}

We consider a general tridimensional system (see Fig.1) where the object of interest is included in a 3D cartesian frame, with coordinates $\boldsymbol{x}=(x, y, z)$. The regular sampling grid of the object is therefore identified by the samples positions $x_{k}$, corresponding also to the center of each basis function $\beta_{k}^{d}$. Then we consider a flat detector, linked with another 2D cartesian frame, with coordinates $\boldsymbol{u}=(u, v)$. The detector acquires the projection with a given orientation denoted $\theta$. The direction of a ray, starting from the X-ray source $S$, orthogonal to the detector plane is identified by the vector $\vec{w}$. The position of $S$ and the vector $\vec{w}$ are directly related to the orientation $\theta$. Let $\mathbf{f}$ be the vector of samples values of the image. The numerical data modelization at orientation $\theta$ is: 


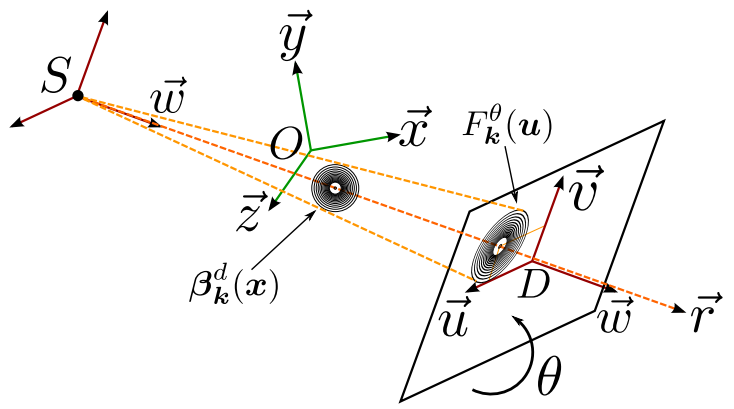

Fig. 1. Cone beam projection scheme of a basis function $\boldsymbol{\beta}_{k}^{d}$ on the detector. $\theta$ denotes the orientation of the detector. The direction of the ray, starting from the source $S(\theta)$, orthogonal to the detector plane is identified by the vector $\vec{w}(\theta)$. The direction of the ray passing through the central position $\boldsymbol{x}_{\boldsymbol{k}}=(x, y, z)$ of $\boldsymbol{\beta}_{\boldsymbol{k}}^{d}$ is identified by the vector $\vec{r}$. The footprint of $\boldsymbol{\beta}_{\boldsymbol{k}}^{d}$ is named $F_{k}^{\theta}$.

$$
\mathbf{g}^{\theta}=\mathbf{R}^{\theta} \cdot \mathbf{c}=\mathbf{R}^{\theta} \cdot\left(\Phi^{-1} \cdot \mathbf{f}\right)
$$

where $\mathbf{R}^{\theta}$ is the projector and $\mathbf{g}^{\theta}$ is the resulting projection. Coefficient $\mathbf{R}_{q k}^{\theta}$ of the matrix $\mathbf{R}^{\theta}$ is the contribution of the voxel function $k$ on the detector pixel $q$. Thus the value $\mathrm{g}_{q}^{\theta}$ of the $q^{\text {th }}$ data element is:

$$
\mathrm{g}_{q}^{\theta}=\sum_{k \in \Omega_{q}^{\theta}} \mathbf{R}_{q k}^{\theta} \cdot \mathrm{c}_{k}
$$

where $\Omega_{q}^{\theta}$ is the set of voxels $k$ impinging the $\theta$-oriented detector pixel $q$. Let $P_{q}: \boldsymbol{u} \mapsto P_{q}(\boldsymbol{u})=\boldsymbol{\beta}^{0}\left(\boldsymbol{u}-\boldsymbol{u}_{q}\right)$ be the $q^{\text {th }}$ detector pixel response, assumed to be a $2 \mathrm{D}$ rectangular pulse, centered at position $\boldsymbol{u}_{q}$. This response is shift-invariant over each detector pixel. Thus:

$$
\mathbf{R}_{q k}^{\theta}=\iint F_{\boldsymbol{k}}^{\theta}(\boldsymbol{u}) \cdot P_{q}(\boldsymbol{u}) d \boldsymbol{u}
$$

$F_{k}^{\theta}$ is the footprint of the basis function $\boldsymbol{\beta}_{\boldsymbol{k}}^{d}$. It is nothing else than the X-ray transform of this function on the $\theta$-oriented detector, along each ray trajectory $\{S(\theta), \vec{r}(\theta, \boldsymbol{u})\}$ crossing it, and impacting the detector plane at the position $\boldsymbol{u}$ (see Fig.1).

$$
F_{\boldsymbol{k}}^{\theta}(\boldsymbol{u})=\int_{\boldsymbol{x} \in\{S(\theta), \vec{r}(\theta, \boldsymbol{u})\}} \boldsymbol{\beta}_{\boldsymbol{k}}^{d}(\boldsymbol{x}) d \boldsymbol{x}
$$

A given projector $\mathbf{R}^{\theta}$ determines the expression of this footprint $F_{k}^{\theta}$. Obviously, $F_{k}^{\theta}$ depends on the chosen basis of functions. Moreover some approximations are often made in the calculation of this footprint and its contribution to detector pixels, in order to lower the computation cost.

Based on staircase voxels, the distance driven projector [1] first considers a plane on which both detector pixels bounds, and the central section of the voxel mostly parallel to this plane, are projected. Then it approximates the footprints with rectangles covering at best the real footprints. Thirdly it determines the overlapping areas of the voxel footprint on the impinged detector pixels footprints. Finally these footprints are used to weight the voxel value, and calculate the contribution of the voxel to each impinged detector pixel. (a)

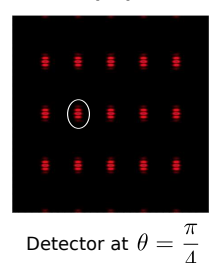

(b)

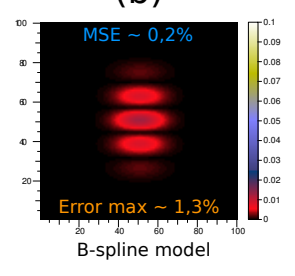

(c)

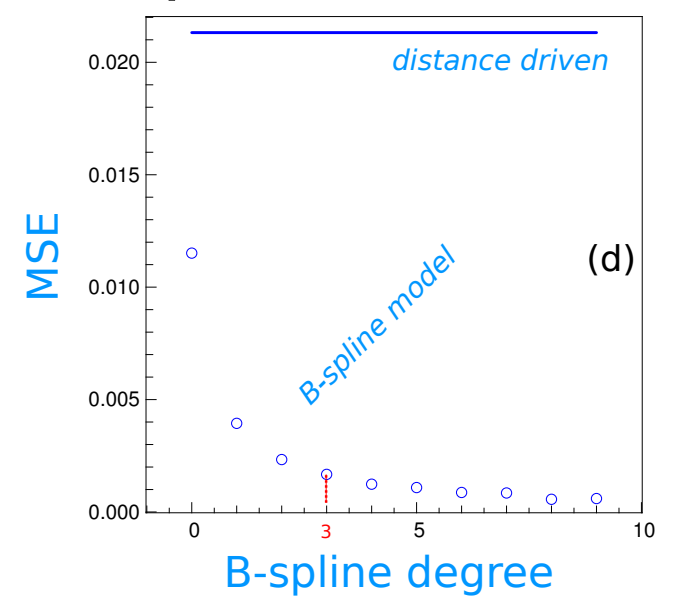

Fig. 2. Comparison of approximation errors for footprints, in parallel beam geometry, for our projector using cubic B-spline, and for the distance driven projector. The detector is in simple rotation around the $\vec{z}$-axis. The worst case orientation of the detector is considered (here it is tilted with an angle of $45^{\circ}$ from the horizontal plane). On (a), (b) and (c), the absolute value of the difference between the true footprint and the model-approximated footprints is represented. Footprints are convolved by the detector pixel response. (a) Illustration of footprints on the detector. (b) B-spline projector with cubic B-splines. (c) Distance driven projector (staircase voxels). (d) shows the evolution of the mean square error as a function of the B-spline degree.

Our approach uses the quasi-isotropy property of B-splines of higher degree, stated in section II-A, to suppose that the footprint is identical whatever the orientation $\theta$. As a result, we first state that the footprint of $\boldsymbol{\beta}_{\boldsymbol{k}}^{d}$, in parallel beam geometry $(\vec{r}(\theta, \boldsymbol{u})=\vec{w}(\theta), \forall \boldsymbol{u})$, is a $n$-1-dimensional B-spline of degree $d$, separable over the detector axis. For the 3D case, this gives:

$$
F_{\boldsymbol{k}}^{\theta}(\boldsymbol{u})=\beta^{d}\left(u-u_{k}\right) \cdot \beta^{d}\left(v-v_{k}\right)
$$

where $\left(u_{k}, v_{k}\right)=\boldsymbol{u}_{\boldsymbol{k}}$ is the position, on the detector, of the projection of the center $\boldsymbol{x}_{\boldsymbol{k}}$ of $\boldsymbol{\beta}_{\boldsymbol{k}}^{d}$. The expression (12) is exact when the direction of parallel beam projection $\vec{w}(\theta)$ is equal to one of the axis directions $\vec{x}, \vec{y}$ or $\vec{z}$; it is the reason why we extend it to all other directions. It also justifies the use of the same spline degree $d$ for the projection (before convolution by the detector pixel response).

Fig.2 displays a quantification of the worst case errors caused by our approximations using cubic B-splines, compared with the distance driven projector's errors, in parallel beam geometry. Our projector proves its better accuracy, while distance driven's approximations look coarser. It also evidences the large decrease of approximation errors, as the B-spline degree increases, as said before. Because a higher degree also means a wider support, the curve Fig.2(d) shows that the use of cubic B-splines is a very good tradeoff between accuracy and compacity. 


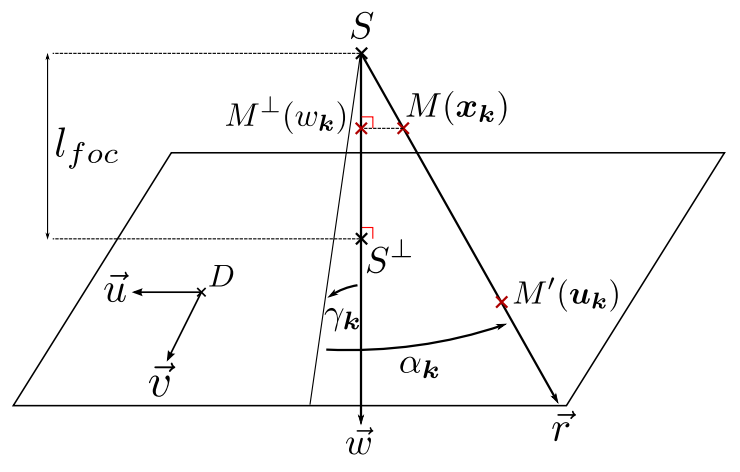

Fig. 3. Scaling parameters in cone beam geometry. $l_{f o c}$ is the focal length of the system (distance between the source point $S$ and and its orthogonal projection $S^{\perp}$ on the detector). $M\left(\boldsymbol{x}_{\boldsymbol{k}}\right)$ is the center of the basis function $\boldsymbol{\beta}_{\boldsymbol{k}}^{d}$. Its cone beam projection on the detector is the point $M^{\prime}\left(\boldsymbol{u}_{\boldsymbol{k}}\right) . M^{\perp}\left(w_{\boldsymbol{k}}\right)$ is the orthogonal projection of $M$ on the straight line $\{S, \vec{w}\}$. Its position $w_{k}$ is used to determine the magnification factor $\Gamma_{S}^{\theta} \cdot \alpha_{k}$ and $\gamma_{\boldsymbol{k}}$ are the cone beam deviation angles related respectively to directions $\vec{u}$ and $\vec{v}$.

In the case of cone beam geometry, the magnification effect has to be taken into account, as well as the distorsion effect depending on the position of the voxel in the field of view (see Fig.3). In order to keep the separability property of the footprint on the detector, these effects are compensated with adapted scaling factors applied to the footprint in (12). For the 3D case, this gives:

$$
F_{\boldsymbol{k}}^{\theta}(\boldsymbol{u})=\beta^{d}\left(\frac{u}{\Gamma_{S}^{\theta} \cdot \delta_{u_{\boldsymbol{k}}}}-u_{\boldsymbol{k}}\right) \cdot \beta^{d}\left(\frac{v}{\Gamma_{S}^{\theta} \cdot \delta_{v_{\boldsymbol{k}}}}-v_{\boldsymbol{k}}\right)
$$

with (see Fig.3):

$$
\Gamma_{S}^{\theta}=\frac{l_{f o c}}{w_{k}}, \quad \delta_{u_{\boldsymbol{k}}}=\frac{1}{\cos \alpha_{\boldsymbol{k}}}, \quad \delta_{v_{\boldsymbol{k}}}=\frac{1}{\cos \gamma_{\boldsymbol{k}}}
$$

$\Gamma_{S}^{\theta}$ is the magnification factor; $\delta_{u_{k}}$ et $\delta_{v_{k}}$ are the distorsion factors.

Fig.4 shows a quantification of the worst case errors due to our approximations using cubic B-splines, compared with the distance driven projector's errors, in cone beam geometry. Errors are enhanced due to approximations made to deal with the geometric effects. However, it brings a confirmation that our projector is more accurate than distance driven.

Therefore our projector involves much less modelization and approximation errors than the distance driven projector. Its only drawback is the increased number of operations necessary to calculate the data values $\mathrm{g}_{q}^{\theta}(9)$, due to the larger footprint of a given voxel. However, cubic B-splines yield sufficient accuracy, inducing a support only 4 times larger than that of the staircase voxel. Let us give the example of a system where the voxels and detector pixels sampling rates are approximately equal. Then let us consider a B-spline of degree $d$. Its support is $s=d+1$ in each direction; thus its footprint impinges approximately $(s+1)^{2}=(d+2)^{2}$ detector pixels. Thus if we compare a cubic voxel $(d=0)$ with a cubic B-spline $(d=3)$, the number of impinged detector pixels for a given voxel, is now multiplied by 6.25 with respect to a cubic voxel based projector such as distance driven. Thus the amount of accuracy is at reasonable increasing computation (a)
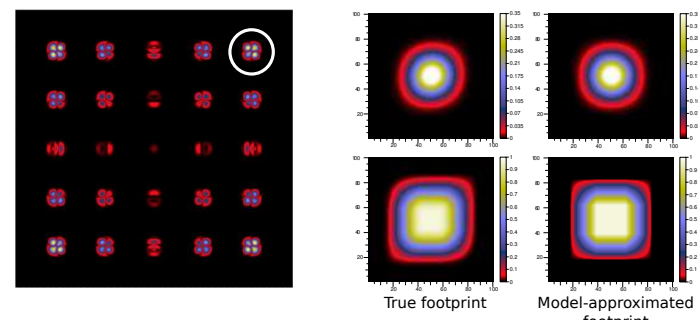

True footprin Model-approximate
footprint Absolute value of the difference

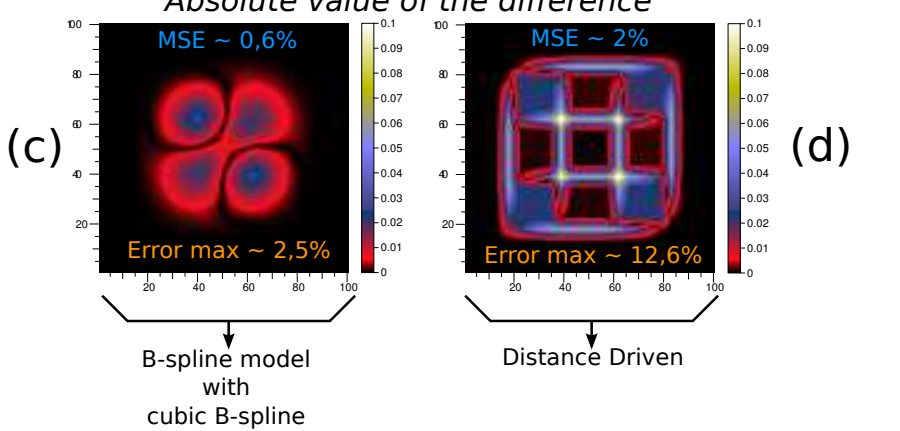

Fig. 4. Comparison of approximation errors of the footprints, in cone beam geometry, for our projector with cubic B-spline, and for the distance driven projector. The detector is in simple rotation around the $\vec{z}$-axis. The worst case orientation of the detector is considered (here it is tilted with an angle of $0^{\circ}$ from the horizontal plane). On (a), (c) and (d), the absolute value of the difference between the true footprint and the model-approximated footprints is visualized. Footprints are convolved by the detector pixel response. (a) Illustration of footprints on the detector. (b) shows, for each projector, the true footprint and the model-approximated footprint. (c) B-spline projector with cubic B-splines. (d) Distance driven projector (staircase voxels).

cost. Moreover our projection scheme, as well as the staircase voxel based approaches, is highly parallelizable, making the computational burden issue possible to address.

The next section will study the gain of our B-spline based projector, when applied to an iterative reconstruction process where only a small number of projections is available.

\section{RESULTS ON 2D FAN BEAM RECONSTRUCTIONS}

A linear detector, linked to the source in the same frame, is considered. The set source-detector rotates around the 2dimensional object of interest, acquiring the projections in a fan beam geometry. The B-spline coefficients of the image c, is reconstructed from the set of projections $\mathbf{g}=\left\{\mathbf{g}^{\theta} \mid \theta \in \Theta\right\}$, where $\Theta$ is the set of projection angles, by minimization of:

$$
\begin{aligned}
\mathbf{c} & =\underset{\hat{\mathbf{c}}}{\operatorname{argmin}} \underbrace{\sum_{\theta \in \Theta}\left\|\mathbf{g}^{\theta}-\mathbf{R}^{\theta} \cdot \hat{\mathbf{c}}\right\|_{\mathbf{W}}^{2}}_{\text {data residuals }}+\underbrace{\mu \cdot \Psi(\boldsymbol{\Phi} \cdot \hat{\mathbf{c}})}_{\text {regularization term }} \\
& =\underset{\hat{\mathbf{c}}}{\operatorname{argmin}} \sum_{\theta \in \Theta}\left(\mathbf{g}^{\theta}-\mathbf{R}^{\theta} \cdot \hat{\mathbf{c}}\right)^{\mathrm{T}} \mathbf{W}\left(\mathbf{g}^{\theta}-\mathbf{R}^{\theta} \cdot \hat{\mathbf{c}}\right)+\mu \cdot \Psi(\boldsymbol{\Phi} \cdot \hat{\mathbf{c}})
\end{aligned}
$$

where $\|\cdot\|_{\mathrm{W}}^{2}$ corresponds to the weighted least squares term. The weighting matrix $\mathbf{W}$ is the inverse of the noise covariance. This matrix is diagonal because we assume the noise to be uncorrelated. $\Psi: \mathbf{f} \mapsto \Psi(\mathbf{f})$ is a regularization operator applied to the image in the samples space. As stated in (4), 
the interpolation operator $\boldsymbol{\Phi}$, which transforms the B-spline coefficients in samples values, can be applied using fast digital filtering operations [15] [16] [17], as well as its inverse. Thus the additional computational burden is negligible.

Here we use a relaxed total variation prior [13]:

$$
\Psi(\mathbf{f})=\sqrt{\left(\frac{\partial \mathrm{f}_{\boldsymbol{k}}}{\partial x}\right)^{2}+\left(\frac{\partial \mathrm{f}_{\boldsymbol{k}}}{\partial y}\right)^{2}+\epsilon^{2}}
$$

The minimization of (15) is performed with a quasi-Newton optimization algorithm: the limited memory Broyden-FletcherGoldfarb-Shanno (L-BFGS) method [12]. From the reconstructed B-spline coefficients $\mathbf{c}$, we obtain the reconstructed image $\mathbf{f}$ by simply applying the operator $\boldsymbol{\Phi}$, to get back in the samples space.

We have reconstructed a $256 \times 256$ Shepp-Logan phantom, from a set of 60 projections with 512 detector pixels, calculated analytically. The sampling rate is the same for both voxels and detector pixels. The reconstructions are performed with both our B-spline based projector, using cubic B-splines, and the distance driven projector, for comparison. The presented reconstructions are obtained from noiseless data first, then from data corrupted by a non-stationary Gaussian noise, with a signal to noise ratio approximately 3000 . Different values of the hyperparameter $\mu$ have been taken in order to look at the effect of the regularization.

Fig.5 displays some reconstructed images. It first illustrates, for reconstructions from noiseless data Fig.5(a), the evolution of the tradeoff between the smoothness of the image and the recovering of details, as a function of the hyperparameter $\mu$. The lower its value, the less smooth the reconstructed image, but at the cost of increasing artifacts, due the lack of projections (ill-posedness of the inverse problem). However our B-spline based projector looks more robust, at decreasing hyperparameter, than distance driven. Then, still for noiseless data Fig.5(b), the best value of the hyperparameter $\mu$ is found, which gives the best qualitative visual quality of the reconstructed image. The criterion is that the image recovers at best the most details, while being sufficiently smooth to avoid noticeable artifacts. Once again, our B-spline based projector leads to a much better image quality than distance driven. The same conclusion can be made in Fig.5(c) with best reconstructions obtained from noisy data, even though a loss of quality is visible with both projectors.

For the noiseless case, the value of the hyperparameter is lower for the B-spline based projector than for the distance driven projector. Hence, the regularization weight being smaller with our method, the data residuals term (15) of the reconstructed image has converged to a lower value. Indeed the convergence of the reconstruction algorithm is a tradeoff between data residuals and prior (regularization). Thus less regularizing allows the solution to converge closer to accordance with the data. The same data residuals can be obtained with distance driven; the corresponding reconstructed image is visualized in (a) for $\mu=0.00001$. Two conclusions can be made from this analysis:

- A lower regularization is needed by the B-spline based projector, to obtain the best image quality, than the distance

\section{distance driven

B-spline model
with
cubic B-splines

(a) Noiseless data: varying hyperparameter
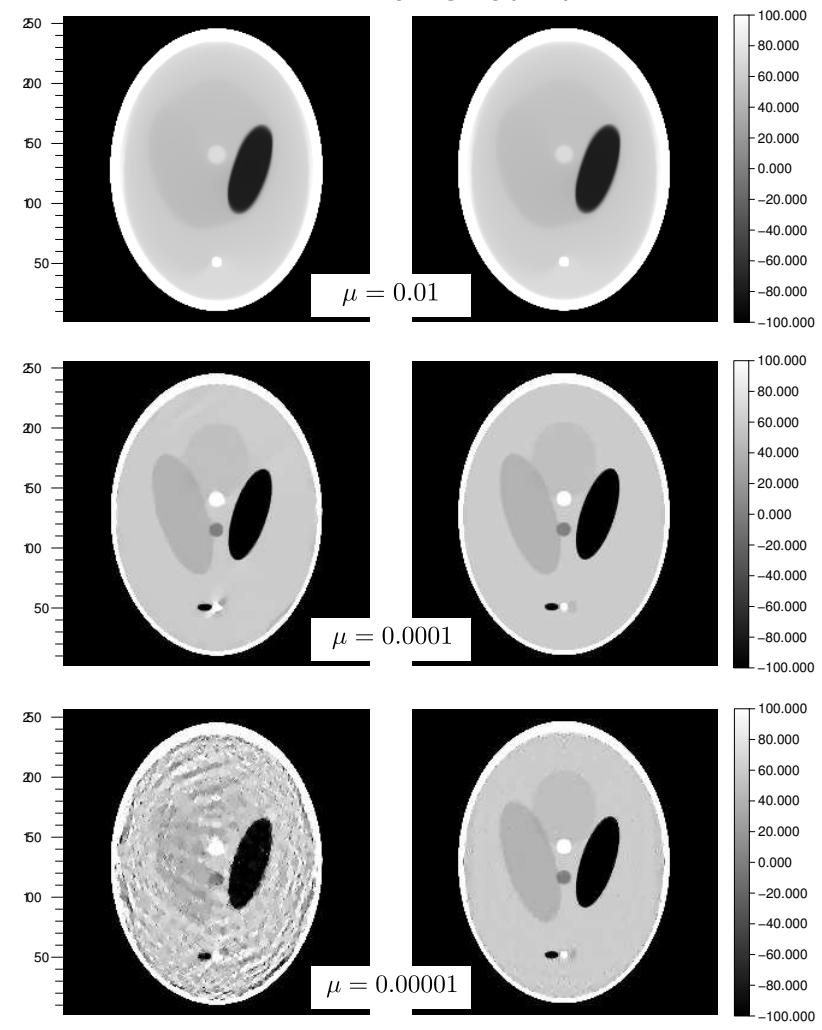

(b) Noiseless data: best hyperparameter

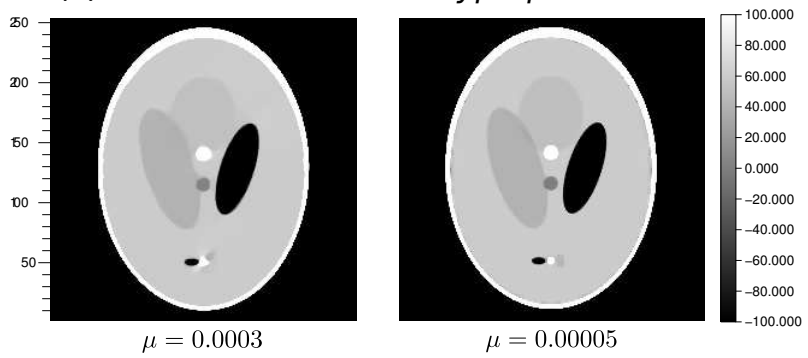

(c) Noisy data $\left(\mathrm{SNR} \sim 3.10^{3}\right)$ : best hyperparameter
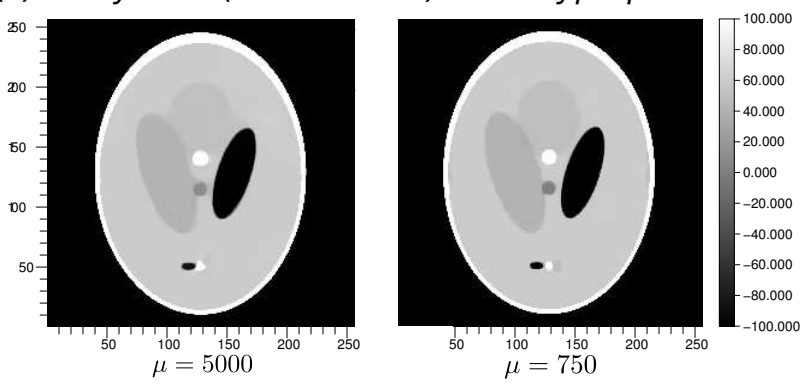

Fig. 5. Reconstructions of a Shepp-Logan phantom $256 \times 256$, from a set of 60 projections with 512 detector pixels, with both the B-spline projector using cubic B-splines and the distance driven projector. Visualization in Hounsfield units. (a) Reconstructions from noiseless data, and for several values of the hyperparameter $\mu$. (b) Reconstructions from noiseless data, obtained with the value of $\mu$ giving the best visual image quality. (c) Reconstructions from noisy data (additional non-stationary Gaussian noise with a signal to noise ratio of about 3000 ), obtained with the value of $\mu$ giving the best visual image quality. 
driven projector.

- For the same accordance with the noiseless data, the Bspline based projector generates a much better image quality than the distance driven projector.

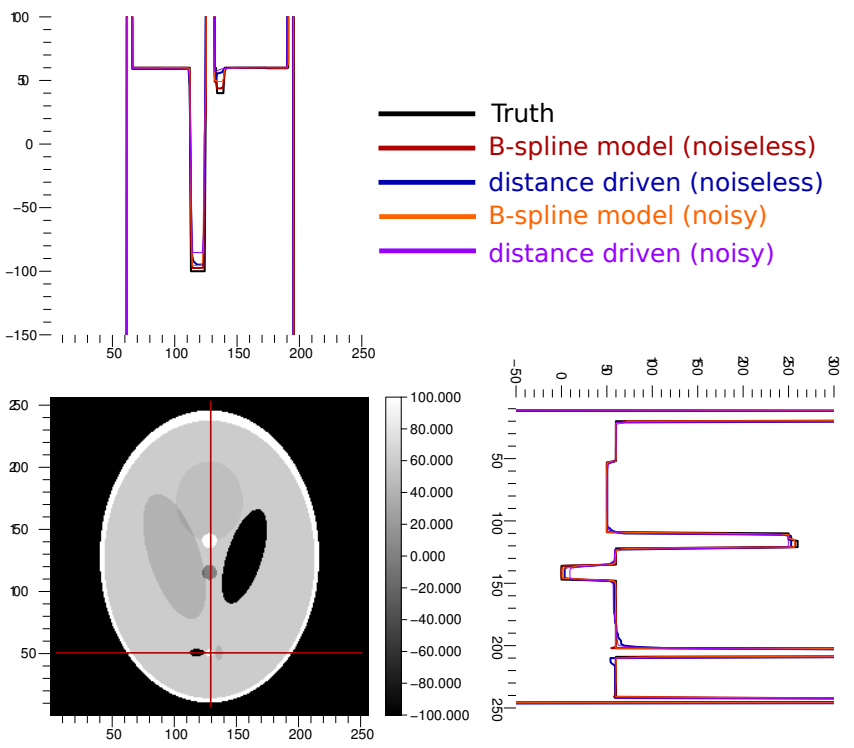

Fig. 6. Profiles of the best reconstructions in Fig.5, from noiseless and noisy data. The cut lines are indicated on the image. In black: the true image. In red $\mathrm{B}$-spline model from noiseless data. In blue: distance driven from noiseless data. In orange: B-spline model from noisy data. In purple: distance driven from noisy data.

Fig.6 confirms the analysis of Fig.5 for the best reconstructed images, from noiseless and noisy data, showing some cut lines of the image. It illustrates the recovering of details, compared with the true image, and proves the better accuracy of our B-spline based projector, with and without noise. We can see, for instance, on the horizontal profile, that the less contrasted of the three small ellipses cut by the line is not recovered when using the distance driven projector, while the use of the B-spline based projector allows to detect it.

Fig.7 shows quantitative results on the reconstructions. The root mean square error (RMSE) has been calculated on 2 regions of interest (ROI) taken on the image. RMSE has then been normalized by the average of the ROI in the true image. The curves Fig.7(a) and Fig.7(b) present the evolution of the RMSE in the 2 ROIs (a given color corresponds to a given ROI), as a function of the hyperparameter $\mu$, for images reconstructed from noiseless (Fig.7(a)) and noisy (Fig.7(b)) data, using both the B-spline based projector using cubic Bsplines (solid curves) and the distance driven projector (dashed curves).

First, the convex shape of each curve illustrates the necessity of precisely tuning $\mu$ to get a good reconstruction which suits the truth and does not increase errors. From these curves, it is possible to approximately find the best hyperparameter determined in Fig.5, taking their minimum. It shows that the RMSE, calculated on well chosen ROIs, seems to be an appropriate metric to evaluate the quality of a reconstructed image.

Secondly, the curves show that, for each ROI, the B-spline based projector's RMSE is always lower than the distance
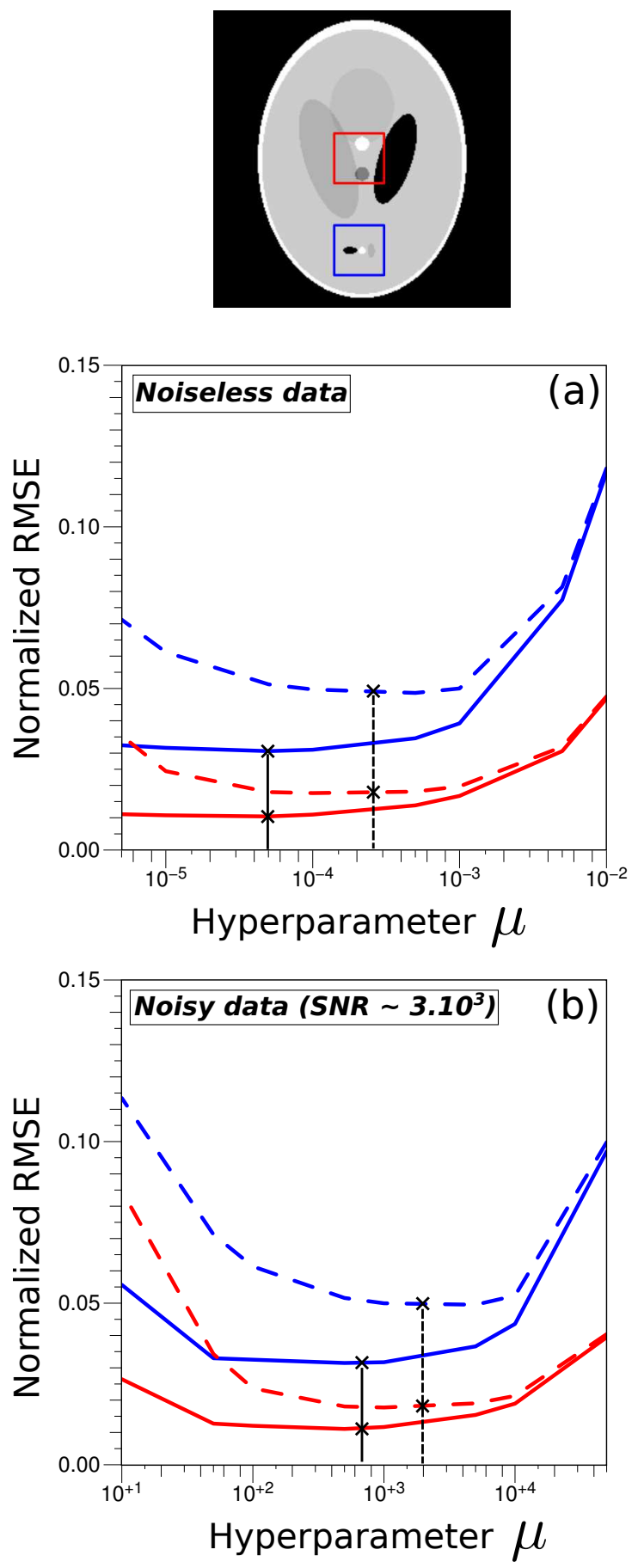

Fig. 7. Root mean square error in 2 regions of interest (ROI) of the reconstructed image, normalized by the average of the ROI, for various values of the hyperparameter $\mu$ (logarithmic scale). The ROIs are indicated on the image, with the corresponding color on the graphs. (a) Noiseless data. (b) Noisy data with SNR $\approx 3000$. The solid curves correspond to reconstructions with the B-spline projector, and the dashed curves correspond to reconstructions with the distance driven projector.

driven projector's RMSE. Thus for this given evaluation metric, our projector shows again the best performances.

\section{CONCLUSION}

In this paper, we have presented a new type of numerical projector for iterative reconstruction in tomography. The pro- 
jector is the numerical modelization of data acquisition; it is based on the mathematical principle of the X-ray transform. We have shown that its accuracy depends on the modelization of the continuous image to be reconstructed; it can allow the reduction of the angular sampling of the data without any loss in the quality of reconstruction. The basis of functions used to discretize the related continuous function on a grid of samples, while keeping an underlying continuous behaviour, is then an essential issue.

We have determined that the use of separable 3D B-splines (compactly supported piecewise polynomials), is the ideal tradeoff between the accuracy of the model and the computation cost, conditioned by the degree of the polynomials. Such a basis of functions is much more adapted to the modelization than the staircase voxels, and easier to manipulate than the blobs.

We have developed a projector, based on the calculation of the footprint of these functions on the detector, and its integration on the impinged pixels. The property of B-splines of tending to the Gaussian curve, with increasing degree (3 or more), has been used to approximate the footprint by a separable B-spline of identical degree on the detector. This makes the calculation of the projections values fast and relatively easy to implement. It also yields less approximation errors than the distance driven projector, which uses staircase voxels.

We have demonstrated, both in a qualitative and quantitative way, the accuracy of our projector based on cubic B-splines, on 2-dimensional regularized iterative reconstructions, from noiseless and noisy simulated data, and from a small number of projections. Reconstructions have shown a substantial gain in image quality compared with reconstructions using the distance driven projector.

Future work will focus on the evaluation of our projector in the 3-dimensional reconstruction case, from real data acquisitions.

\section{REFERENCES}

[1] B. DeMan and S. Basu. Distance driven projection and backprojection in three dimensions. Physics in Medicine and Biology, 2004.

[2] J.A. Fessler. Iterative methods for image reconstruction. IEEE International Symposium on Biomedical Imaging Tutorial, Arlington Virginia, 2006.

[3] Y. Long, J.A. Fessler and J.M. Balter. 3D Forward and Back-Projection for X-Ray CT Using Separable Footprints. 10th Intl. Mtg. on Fully 3D Im. Rec. in Rad. and Nuc. Med., 2009.

[4] S. Horbelt, M. Liebling and M. Unser. Discretization of the Radon transform and of its inverse by spline convolutions. IEEE Transactions on medical imaging, 2002.

[5] P.M. Joseph. An improved algorithm for reprojecting rays through pixel images. IEEE Transactions on Medical Imaging, 1982.

[6] M. Slaney and A. Kak. Principles of computerized tomographic imaging. SIAM, Philadelphia, 1988.

[7] R.M. Lewitt. Multidimensional digital image representations using generalized Kaiser-Bessel window functions. JOSA A, 1990.

[8] R.M. Lewitt. Alternatives to voxels for image representation in iterative reconstruction algorithms. Physics in Medicine and Biology, 1992.

[9] S. Matej and R.M. Lewitt. Image representation and tomographic reconstruction using spherically symmetric volume elements. IEEE Transactions on Medical Imaging, 1993.

[10] S. Matej and R.M. Lewitt. Efficient 3D grids for image reconstruction using spherically-symmetric volume elements. IEEE Transactions on Medical Imaging, 1995.
[11] S. Matej and R.M. Lewitt. Practical considerations for 3D image reconstruction using spherically symmetric volume elements. IEEE Transactions on Medical Imaging, 1996.

[12] J. Nocedal. Updating quasi-Newton matrices with limited storage. Mathematics of computation, 1980.

[13] L.I. Rudin, S. Osher and E. Fatemi. Nonlinear total variation based noise removal algorithms. Physica D: Nonlinear Phenomena, 1992.

[14] P. Thévenaz, T. Blu and M. Unser. Interpolation Revisited. IEEE Transactions on Medical Imaging, 2000.

[15] M. Unser., A. Aldroubi and M. Eden. B-spline signal processing: Part I-Theory. IEEE Transactions on Signal Processing, 1993.

[16] M. Unser., A. Aldroubi and M. Eden. B-spline signal processing: Part II-Efficient design and applications. IEEE Transactions on Signal Processing, 1993.

[17] M. Unser. Splines: A perfect fit for signal and image processing. IEEE Signal Processing Mag., 1999.

[18] M. Unser. Sampling-50 years after Shannon. Proceedings of the IEEE, 2000. 